\title{
To Boldly Go: Feedback as Digital, Multimodal Dialogue
}

\author{
James Lamb \\ Centre for Research in Digital Education, Moray House School of Education, University of Edinburgh, \\ Edinburgh EH8 8AQ, UK; j.i.lamb@sms.ed.ac.uk
}

Received: 22 May 2018; Accepted: 22 August 2018; Published: 27 August 2018

\begin{abstract}
This article is concerned with digital, multimodal feedback that supports learning and assessment within education. Drawing on the research literature alongside a case study from a postgraduate program in digital education, I argue that approaching feedback as an ongoing dialogue presented in richly multimodal and digital form can support opportunities for learning that are imaginative, critical, and in-tune with our increasingly digitally-mediated society. Using the examples of a reflective blogging exercise and an assignment built in the Second Life virtual world, I demonstrate how the tutor's emphasis on providing feedback in multimodal form, alongside more conventional print-based approaches, inspired and emboldened students towards the creation of apt and sophisticated coursework. At the same time, the crafting of multimodal feedback carries resource implications and can sit uncomfortably with some deep-rooted assumptions around language-based representations of academic knowledge. This article should be seen in the context of a growing pedagogic and institutional interest in feedback around assessment, alongside the emergence of new ways of communicating and consuming academic content in richly multimodal ways. In this setting, multimodality, technology, and interaction refers to the digitally-mediated dialogue that takes place between the student and tutor around assessment.
\end{abstract}

Keywords: multimodality; feedback; assessment; dialogue; digital

\section{Introduction}

When I logged into the learning management system to retrieve Graeme's final assignment, I expected to find a pdf or link to a web essay or video. Instead, the submission dropbox displayed a set of coordinates in a format I recognized to be from the Second Life virtual world, one of the online spaces we had used during the Introduction to Digital Environments for Learning course. After pasting the coordinates into the destination box in Second Life, I was transported, in the guise of my avatar Jimmi, to a coastal location within the Virtual University of Edinburgh. After an awkward landing, I surveyed my surroundings and observed a sign splitting the ocean and pointing in the direction of the seabed:

'To Boldly Go! A Quest for the Holy Trinity of Presence, Immersion and Flow within Education Virtual CPD Spaces'

Following the direction of the sign, I guided Jimmi below the surface of the ocean and navigated a path towards a training room, with a projection screen and seating for one. Reclining into my beanbag, I hovered the cursor over the screen, which prompted an invitation to watch a video of Graeme reflecting on the potentialities of virtual worlds within continuing professional development (see Figure 1). 


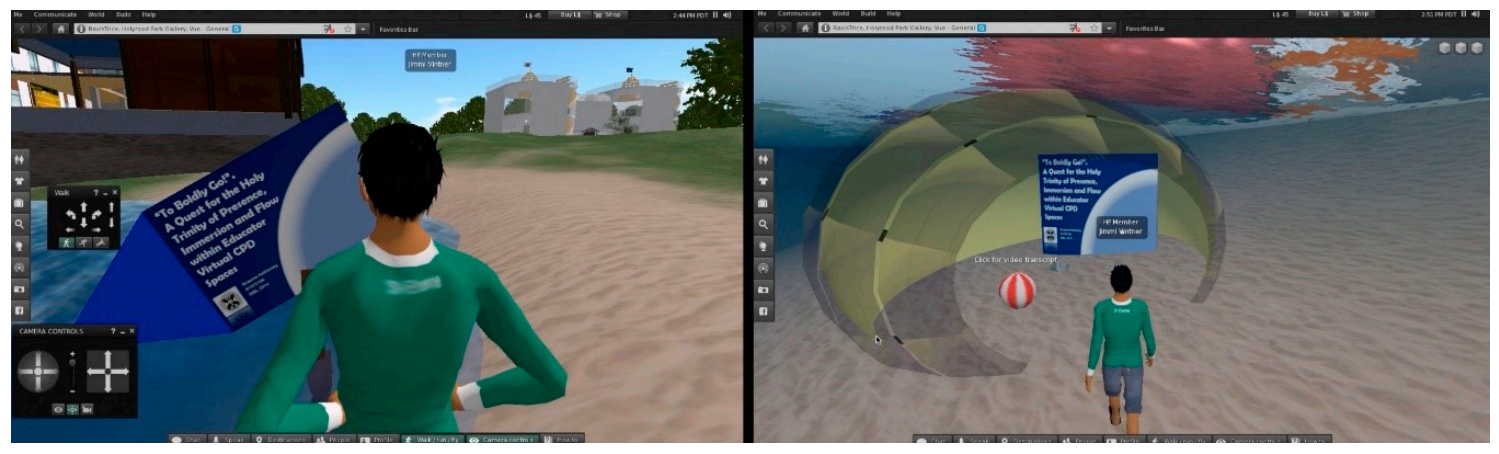

Figure 1. Navigating my way to Graeme's Second Life assignment. Image by the author.

\section{Context}

This article is situated at the intersection between multimodality, digital education, and the feedback that takes place around assessment. After discussing the key themes from the relevant literatures, these ideas are explored and illustrated using a case study that draws on my own experience of multimodal feedback as a tutor in the MSc in Digital Education program at the University of Edinburgh. This is a fully online postgraduate program at a research-led institution that challenges students to consider how teaching and learning is affected by the pedagogic and societal shift to the digital. In the pages that follow, I draw on my experiences as a course tutor, alongside the literature around multimodality and feedback, to discuss how Graeme's Second Life assignment and other similarly imaginative examples of coursework, were supported by digitally-mediated, multimodal dialogue. The increasingly digital nature of education and society presents new and exciting opportunities for communicating, consuming, and constructing academic knowledge in multimodal ways [1]. Where language has for centuries dominated representational practices in and around the classroom [2], the proliferation of digital devices and social media spaces across campus and society encourages and allows students and teachers to engage in dialogue across words, images, sounds and the full range of meaning-carrying resources [3]. The central argument of this article is that, subject to context, digital multimodal dialogue can provide an effective, exciting, and apt way of supporting learning around assessment.

Across the last two decades, there has been considerable interest in the relationship between education and multimodality within our increasingly digitally and visually-mediated society. An important part of this discourse is whether and how assessment practices should place a greater emphasis on multimodal meaning-making in response to technological advances [4], to better align with the complex and multi-faceted literacy skills of students [5], to equip graduates with the creativity needed in the workplace [6], to meet the challenges of a global economy [7], and to respond to the already digital and multimodal nature of teaching [8], amongst other reasons. When feedback practices are understood to be deeply implicated in the nature of assessment, it is surprising, then, that the critical interest in the relationship between multimodality and assessment has rarely extended to consider the implications of multimodal feedback. By making the case for digital multimodal dialogue, this article makes explicit the connection between technology, multimodality, feedback, and assessment.

\section{Scope and Structure of this Article}

Although this article considers themes from the research literature through the example of a postgraduate-level course in education, in common with many of the principles of feedback, the ideas I present around the possibilities and challenges of digital, multimodal dialogue should resonate with teachers and researchers concerned with secondary school, college, and undergraduate education. At the same time, while I discuss digital, multimodal dialogue in the setting of an education program, I believe my central arguments could equally apply across the humanities and social sciences, alongside 
other courses and contexts where the printed word has tended to hold sway. In the first half of this article, I draw on the literature to account for the critical, pedagogic, and institutional significance of feedback around assessment before accounting for the growing critical interest in multimodal assessment. This is followed by a discussion of the small body of work specifically interested in the relationship between multimodality and feedback. From there my attention turns to the case study introduced in the opening to this article, where I describe and discuss digital, multimodal dialogue in practice, before reflecting on the implications for teachers, including my own experiences as a tutor. The article concludes by reiterating the case for digital, multimodal dialogue, albeit dependent on context and in conjunction with more conventional feedback strategies. Before all of this, however, I wish to set out my position around feedback, digital technology, and multimodality.

\section{Feedback}

In light of the interest of this special issue, I have found it helpful to combine two related conceptualizations of feedback. First, I have been drawn towards the work of Hatzipanagos and Warburton [9], where they propose ongoing technologically-mediated dialogue as a strategy for supporting learning around assessment. Hatzipanagos and Warburton take the position that the social dimension of emergent technologies presents opportunities for rich dialogue that can support formative assessment and ongoing feedback. Also useful has been the concept of feedforward (advanced, for instance, by Hounsell et al. [10]) where students are provided with correctional advice and guidance during the process of composition, rather than beyond the submission of coursework. In this way feedback becomes an evolving, digital conversation between student and tutor that promotes regular opportunities for encouragement and discussion around learning and assessment. As will become apparent in the pages that follow, I have also drawn on the work of David Boud, Valerie Shute, and other key voices in the field in order to frame the nature of feedback within increasingly digital and multimodal learning environments. Finally, while acknowledging that recent conceptualizations of feedback have moved beyond placing emphasis upon the exchange of information between student and tutor, for instance, to highlight the potentialities of peer, self-generated, and automated feedback, my interest within this article is in the dialogue between learner and teacher.

\section{Technology}

The intimate relationship between education and technology can be traced at least as far back as the moments when professors, scribers, and rubricators first assembled around the Gutenberg presses [2], prompting the swift reproduction and distribution of anatomical, botanical, and naturalist knowledge [11]. The interest of this article, however, is in the contemporary screen-based technologies that encourage and allow for the communication of meaning across a range of modes. Therefore, where I refer to technology in the pages that follow, I am concerned with digital devices (such as the smartphone and computer), applications (examples of software), and platforms (online environments including learning management systems and social media spaces) that are increasingly integral to the educational activity that takes place within and beyond the classroom. I reject, however, the instrumentalist tendency to regard digital resources as tools to deliver learning [12], or an essentialist position whereby technology is seen to be the dominant driver of pedagogic change [13]. Drawing on conceptual work around sociomateriality in education [14-16], I take the position that feedback is performed through an assemblage of technological, human, institutional, and other material interests and opportunities. The value of a sociomaterial approach to education research, according to Fenwick et al. [14], is through the way that it draws attention to the broader range of human and non-human interests, resources, and opportunities that shape what takes place in and beyond the classroom. In this way multimodal dialogue is negotiated through a sociomaterial assemblage that combines the interests and abilities of teacher and student, but also the potentialities and problems of digital devices, the availability of resources and the pressure or impetus of institutional strategy. 


\section{Multimodality}

The discussion that follows is broadly informed by an understanding of multimodality associated with the work of Kress \& Van Leewen [1] and others who look toward a social semiotic tradition. At the same time, my interest in the phenomenon of multimodal feedback is sufficiently broad, I believe, to transcend different multimodal traditions, whilst being sufficiently accessible to those approaching this article with a primary interest in feedback, learning technology, or education more generally. Looking particularly to articulations of multimodality by Kress [17] and by Jewitt [3], I take the position that all communicational acts are multimodal, and that the way we interpret meaning is shaped by the particular selection and configuration of modes. Also useful in informing my thinking has been multimodality's openness to the full range of resources that have the potential to convey meaning, whilst recognizing that what we define as a mode is socially and culturally shaped. At the same time, the critical interest in multimodality and multimodal learning is closely tied to the increasingly digital nature of society and education [18] that provides the backdrop to this article.

If we accept that all communicational acts are multimodal in the way that they depend on an orchestration of semiotic material, then the question emerges of whether feedback can be anything other than multimodal. A common feature of the literature that has looked to the relationship between multimodality and education has been an interest in introducing or emphasizing multimodal ways of teaching and learning presumedly alongside or in place of "monomodal" [19] or overtly-language-based approaches. The apparent ambiguity here exists through the impossibility of a single taxonomy of modes when meaning is socially and culturally situated [1]. Applied to the context of feedback around assessment, the typical typed feedback pro forma could be understood either as monomodal in that the communication of meaning depends entirely on the mode of language, or alternatively multimodal through the selection of font, color, spacing, and layout, all of which convey meaning in juxtaposition with the content of the words on the page or screen. We might navigate our way out of this conceptual conundrum through the concept of modal density advanced by Sigrid Norris [20]. Modal density, according to Norris, can be used to describe the complexity of the representational act, for instance, when it depends on a broad range of semiotic material. Taking the example of a video recording where a tutor discusses the relative strengths and weaknesses of an assignment, there will be a broad and complex entanglement of modes associated with verbal communication, including the use of language, tone, tempo, and volume, as well as non-verbal communication including gesture, gaze, posture, eye contact, and so on [3]. If the teacher's spoken comments were instead transferred to a conventional feedback pro forma there would almost certainly be a narrower, simpler and therefore less dense orchestration of modes. Therefore, the feedback video would demonstrate greater modal density than the typed version. In this way, Norris's modal density allows for the varying breadth of semiotic material within a representational act while remaining true to the conceptual principle that all communication is multimodal by varying degree. Where "dense" carries connotations of impenetrability or murkiness within compositional and educational contexts, I instead use "multimodal richness" to account for representational acts where knowledge is conveyed across a broad range of resources. It is worth making clear, however, that digitally-mediated feedback is not, by definition, richly multimodal; on the contrary, many examples of peer, automated, and self-generated feedback systems, predominantly or entirely, depend on comments or guidance expressed through words-in-isolation. Neither is richly multimodal feedback restricted to the digital form; such an idea is immediately dispelled by watching how the classroom teacher offers correctional advice or guidance through a varied assemblage of spoken language and silence, but also using posture, gesture, eye contact, and beyond. Multimodal dialogue within this article, however, specifically refers to digitally-mediated communicational acts where meaning is conveyed across a broad range of semiotic material. 


\section{The Rationale Supporting Multimodal Assessment}

When assessment and feedback are recognized to be intimately linked, it is helpful to briefly consider the rationale for paying closer attention to multimodality within the design of coursework assignments and similar summative work. The growing interest in the relationship between multimodality and assessment is presented against the backdrop of an increasingly digital society. It is a feature of the literature that discussion frequently opens by identifying the need for assessment practice to evolve in order to keep up with the changes taking place beyond the boundaries of the classroom and campus. Mills argues that multimodal assessment needs to adapt in order to better reflect the "life validity" of social contexts that exist beyond schools [21] (p. 262), while Miller et al. [22] point towards the potential for video composition to more authentically reflect the reality experienced outside of the classroom. This is a reality, the literature repeatedly affirms, that is being profoundly shaped by the proliferation of digital content and devices, which in turn present an ever-growing array of ways of consuming and communicating knowledge. The evolving nature of knowledge construction is neatly captured by Archer in her discussion of the challenges that multimodality poses to the teaching of writing when she describes the "changing representational landscape" [23] (p. 387) in which teaching, learning, and assessment finds itself situated. As communicational practices across education and society have evolved, assessment has sometimes been reluctant to adapt to the way that literacy has been reconfigured by the screen, according to Jewitt [24]. A further dimension in the rationale for multimodal assessment is the need for better alignment with the evolving literacy practices of students $[6,25,26]$ who have grown up surrounded by digital devices and resources that lend themselves to the communication and consumption of content across a range of modes. In the move towards multimodal assessment we should be cautious, however, to conflate technical proficiency with the ability to work critically with digital resources, according to Carpenter [5]. Meanwhile in a large-scale study into examples of multimodal work produced by school students, Kimber \& Wyatt-Smith [27] identified varying levels of technical ability, which usefully discourages us from assuming a homogenous body of learners with a high level of technical sophistication. Nevertheless, the literature offers a broad rationale for multimodal assessment's capacity to better reflect the changing representational landscape [23] and the communication practices and needs of learners [22], while meeting the challenges of a global economy [7] and the creativity needed in the modern work place [27]. From a purely pedagogic perspective, however, the case is perhaps best made by Jewitt [24] who argues that when teaching practices are already increasingly multimodal, assessment needs to evolve in order to avoid missing out on what has been learned in the classroom.

\section{Towards Digital, Multimodal Feedback}

Over the last decade a combination of pressures and opportunities have contributed toward a considerable interest in feedback amongst teachers, researchers, learning technologists, and education managers. This is reflected in an extensive body of published research, alongside conferences, institutional strategies and "best practice" guides on the subject of feedback. In the U.K. context, a heightened attention towards feedback has resulted in part through the marketisation of higher education, combined with a culture of performativity that has placed scrutiny on the frequency and quality of guidance that students are entitled to receive. Feedback has become an instrument for ranking universities in the league tables produced by broadsheet newspapers, as well as a prominent theme in the National Student Survey (NSS) where final-year undergraduates are invited to comment on the quality of their higher education experience [28]. When the NSS website advertises the potential for its gathered data to influence the thinking of prospective university applicants, and results suggest that students are dissatisfied with the quality of what is being provided [29], feedback takes on a strategic importance than can sharpen the minds of education managers who might not otherwise have been so attentive to the frequency or focus of dialogue between students and tutors.

Whether driven by league table performance or wider pedagogic interest, there has been a significant change in thinking around the timing and frequency of feedback. Where feedback has 
traditionally described the comments assigned to a piece of summative coursework, typically at or beyond the end of a course of study, there has been a broad recognition of the need to provide students with guidance at an earlier stage. The emphasis on feedforward rather than feedback [10] means that the teacher's comment are more timely as they enable the student to take corrective action that will improve the quality of the assignment she subsequently submits to be marked and graded. The earlier provision of feedback (or feedforward) can be provided in a number of ways, for instance, through the use of formative assessment exercises where students are able to gauge their progress and understanding in a low-stakes setting [10]. In her discussion of formative assessment Shute [30] refers to "information communicated to the learner that is intended to modify his or her thinking or behavior for the purpose of improved learning" (p. 152) through the potential to "increase student knowledge, skills and understanding" (p. 156). More generally, there has been an emphasis on providing regular opportunities for students to engage in dialogue with teachers, with the aim of providing a clearer understanding of their progress and what will be expected within summative assessment [9]. At least in the context of U.K. higher education, providing more regular opportunities for interaction has proven to be problematic, however, against a backdrop of growing student numbers without a comparable increase in teaching staff. The growth in student numbers and scrutiny of feedback practices has coincided with a longer period of educational change where opportunities for formative assessment have diminished, according to Boud and Molloy [28], for instance, as the use of regular short essays in higher education has been replaced by a series of summative coursework assignments. Declining opportunities for formative feedback have reduced the regularity with which students have the opportunity to get a sense of their own understanding and performance, whilst limiting opportunities for low-stakes experimentation $[10,31]$ that is seen as a highly important part of supporting learning and building student self-efficacy [30,32].

In light of the challenge of generating further opportunities for timely and meaningful feedback, attention has turned to the possibility of using technology to provide new forms of dialogue and interaction around learning and assessment. This has included the use of personal response systems as part of a "flipped classroom" approach where students generate feedback on their understanding by responding to quiz exercises in class [33]. Meanwhile, applications such as PeerWise enable students to collect feedback as they respond to multiple choice questions created by their peers [34]. Elsewhere, simpler automated quiz activities are now a standard feature of learning management systems, sometimes placed alongside discussion boards where students can discuss their answers with peers or more generally gain feedback through conversation with students and staff [9]. Other forms of digitally-mediated feedback include screencasts [35] as well as annotation software, audio commentary and systems for the adaptive release of teacher comments [36].

The examples described here are typical of the wider discourse in the way that emergent digital technologies are employed to support or enhance mostly conventional feedback approaches, or as a way of efficiently increasing the amount of dialogue around assessment and learning, whether on account of growing student numbers or an institutional concern with student satisfaction and benchmarking (or a combination of all these reasons). The relationship between feedback and technology has been differently explored, however, within the field of learning analytics where it has been of considerable critical interest [37]. The value of a learning analytics approach to feedback practice is set out by Gasevic et al. [38] when they argue that through an analytical appreciation of learning processes and learner abilities, teachers might "identify weak points in the learning activities performed by their students; topics the students have struggled with, and provide instructive and process related feedback on how to improve their learning" (p. 66). The potential of learning analytics, educational data mining, and the use of algorithms lies, it is argued, in their capacity to support critical and context-sensitive approaches to feedback that seek to take account of what is distinct about the particular assessment setting. Furthermore, educational data mining and learning analytics, according to Pardo [39], can be used to address a prevailing disconnect between theoretical discussions of feedback and evidence of resultant improvements in academic performance. In place of feedback models that inadequately 
demonstrate how the likes of feedback loops translate to learning gains or improved understanding, Pardo proposes a framework that sets out an explicit link between educational analytics and the potentialities of the algorithm with feedback and cognitive processes. While Pardo's work is set out at a conceptual level, it would seem able to frame the particular complexities of digital, multimodal dialogue through the way that it "takes into account other important mediating factors such as the role of the instructor and the need to consider changes in knowledge, beliefs, attitudes, strategies and tactics in the learner as the ultimate goal of the process" (p. 437). Learning analytics and educational data mining have also become an important part of the discussion around learning at scale, particularly in light of the need to provide targeted and timely feedback to a growing body of students. As students interact with educational technologies, according to Pardo et al. [40], they generate data that can offer a picture of their personal learning performance and needs, followed by the tailoring of personal feedback. In the setting of an undergraduate engineering course, Pardo et al. describe how an approach that combined instructor knowledge with digital traces of student interaction around technology allowed for the generation of regular and personalized e-mail feedback messages that met with a positive response from learners and provided evidence of improved academic performance within mid-term examinations. The potential for the complex workings of code and algorithm to support feedback and understanding is also shown in Shute \& Ventura's work around video game-based learning [41]. Proposing the concept of "Stealth Assessment," Shute \& Ventura argue that game-playing supports feedback around learning in a range of ways, for instance, through the generation of real-time information that generates hints and alters the difficulty level of an activity in response to the student's particular needs-in-the-moment. Crucially, feedback generated in this way potentially offers the considerable advantage of being timely, personalized, and ongoing without providing the type of workload burden that can be prohibitive for teaching staff with competing responsibilities and time pressures.

\section{Review of the Literature}

The discussion of the literature that follows draws attention to work that has taken a critical interest in the multimodal character of feedback in educational contexts. Beginning in the setting of the higher education language classroom, Sadoux [36] discusses a pilot project where she used "generic online multimodal feedback" to encourage higher levels of student engagement. In light of the burden that feedback can present teachers with, Sadoux replaced individualized comments with a PowerPoint presentation and accompanying narration (edited into a video) that addressed broad issues around the assignment, whilst inviting students to contact her with specific follow-up queries. Drawing on work in cognitive learning theory, Sadoux argues that multimodal feedback enables the student to process information through different memory systems, "thus improving performance and reducing cognitive overload" (p. 89). Sadoux cites email correspondence with students and viewing statistics to suggest a positive reception towards this generic multimodal feedback.

Where Sadoux's pilot study was mostly concerned with delivering feedback in response to an assessment exercise, Campbell \& Feldman [42] place a greater emphasis on multimodal feedback as a form of technological exchange during the process of composition. In their discussion of the power of multimodal feedback in the school English classroom, Campbell and Feldman describe how they were motivated by a desire to explore whether technology could provide more "streamlined" and "efficient" ways of providing feedback around the writing process. Defining multimodal feedback as "a combination of audio, video and visual media" [42] (p. 1), Campbell and Feldman describe how providing students with comments on their written work at a formative stage resulted in prompt revisions, an impact they argue to be much more suggestive of learning than assigning a grade or circling an achieved outcome on an assessment rubric. Of note, Campbell and Feldman describe how this multimodal and iterative approach resulted in the improved compositional performance of a student who previously experienced difficulty in his writing. There is a parallel here with critical work that has demonstrated how an emphasis on multimodality within assessment more 
generally has proven to be transformative for learners experiencing some form of educational or social disadvantage $[26,43]$. Although it is not the interest of this article, there is the intriguing possibility that digitally-mediated multimodal feedback might have a role to play in enabling students from non-traditional backgrounds or positions of educational disadvantage to better understand and put into action comments and advice from teachers.

In contrast to the generic emphasis of multimodal feedback proposed by Sadoux [36], Campbell and Feldman [42] describe how the use of video conferencing, audio recordings, and annotation software supported individualized feedback that led to improved understanding of content, while building relationships between staff and students. Particularly important to Campbell and Feldman was the potential for these multimodal approaches to generate feedback that was timely, which is firmly established as central to "best practice" around feedback [10,44]. There is a need, however, to establish the extent to which the observed success of multimodal feedback depended on the particular selection and configuration of semiotic resources, or whether it was attributable to the dialogic or timely nature of the approach more generally. Therefore, where Campbell and Feldman describe how multimodal feedback "allows the student to pause and revise and play again" and to "revisit the feedback at a later time" through the ease with which "video and audio files are archivable and readily available" [42] (p. 2), we might see a parallel with the student who casts an eye over an annotated essay or printed feedback sheet before filing it away for future use. At the same time, the accessibility, longevity, and portability of audio or video feedback has more to do with its digital form more generally, rather than its multimodal make-up. In this and other examples across the literature, there is a need to better explain what it is about an audio commentary (or other approach) that makes it more overtly multimodal compared to the same guidance being offered in a conventional (non-digital) way, for instance, through spoken conversation in the classroom. More generally, while the different pieces of research described in this review advertise a specific interest in multimodality, they would sometimes benefit from taking a position on what multimodality is: in the absence of this type of conceptual framing there is a tendency to under-consider what is multimodal about a particular form of feedback, giving the impression that "multimedia" might be a better fit for the approach. An exception here would be Mathisen's discussion of video feedback around written composition where he raises the question of how the teacher might "optimize the interplay between the visual and verbal" [11] (p. 111) within the video format, or what we might describe from a multimodal perspective as questions around the particular configuration and prominence of modes within a communicational act.

Where the research by Sadoux [36] and by Campbell and Feldman [42] investigated multimodal feedback in a single learning context, Mathisen studied the use of screen capture videos across eight higher education subjects. Like Campbell and Feldman, Mathisen observed that multimodal feedback would inspire and motivate students in their writing, while establishing closer working relationships with teachers. From the teacher's perspective meanwhile, the simplicity of the screen format supported higher levels of precision and quality in the feedback they were able to provide. While highlighting the possibilities for enhanced learning and classroom relations, Mathisen calls for a nuanced approach when considering the success of multimodal feedback. Mathisen asks, for instance, whether the video format might be particularly suited to the interests of some learners, and within particular academic disciplines, but less welcome or suitable in others. While Carless and Boud are amongst those who have acknowledged that students respond positively to digitally-mediated feedback, they also highlight the need to consider the importance of "affect," which they describe as the learner's "feelings, emotions and attitudes" [29] (p. 3). When a single of group students enter a classroom with varying abilities, interests, and histories, Mathisen, along with Carless and Boud, helpfully remind us to be alert to the value of personalizing feedback and consider varying feedback literacy and wider background. The importance of considering affect and recognizing the complexity of student needs over a digitally-driven one-size-fits all approach is also recognized by Elola and Oskoz [45] in their discussion of the potential for multimodal feedback to support second language learning. Rather than framing discussion in terms of old versus new (which is a feature of the 
discourse around multimodality and education), Elola and Oskoz point towards the complementarity of digitally-mediated multimodal feedback and more conventional printed-language approaches. Drawing on their study of four students in an advanced level Spanish writing course, Elola and Oskoz observed that students preferred a multimodal commentary for broader issues such as content, however favored more traditional typed feedback when it came to the particular form of their writing. The message here is that in considering the potentialities of multimodal feedback we should be open to considering new digitally-mediated dialogue as part of a wider repertoire of feedback methods.

Any attempt to evaluate the value of multimodal feedback needs to take account of the wider entanglement of human, material, technological, and political interests and opportunities that contribute towards educational practice [12,14-16]. In their research around student perceptions towards digitally recorded multimodal feedback, Philips et al. [46] call for greater attention to the influence of secondary factors including preferred learning style, access to technology, institutional assessment policies, and so on. In a similar vein, Mathisen argues that in judging the success of multimodal feedback strategies, we need to pay attention to wider educational context and circumstances, including "the interaction between technology and the particular subject's distinctive character, existing educational and advisement traditions as well as the teacher and student's digital literacy" [12] (p. 100). When there can be a tendency to consider feedback as a closed exchange between tutor and student, we can usefully extend the position taken by Mathisen and Philips et al. to acknowledge the wider body of human and non-human materialities that are implicated in education practices [14]. We are reminded here that the interaction between student and tutors always exists as part of wider sociomaterial assemblage of interests, opportunities, resources, and pressures, including the availability and capability of digital technology, institutional pressure, deadlines, and so on. Mathisen goes on to challenge instrumentalist assumptions which connect the introduction of technological tools with delivered learning success. In a similar vein, Philips et al. argue that, "using technology such as video is not a silicon bullet to improving feedback" [46] (p. 514); instead, where multimodal feedback is observed to be successful, we need to unpick whether the value it brought to student learning is attributable towards a particular orchestration of semiotic material, or whether it rested with a feedback strategy that was more widely dialogic, timely, or demonstrative of other strategies associated with improved learning and performance around assessment.

Where the examples described here approach feedback from the conventional perspective of an exchange between student and tutor, recent research by Hung [47] turned attention to the role of multimodal video feedback between students. Situated within the EFL (English as a Foreign Language) classroom, Hung analyzed learner reflections, interview transcripts, and questionnaire data from 60 students to investigate experiences and attitudes towards video feedback in place of more traditional written approaches. Amongst the key themes to emerge from Hung's research was the overall positive response of learners, particularly towards more personalized feedback generated by their peers via video. This was accompanied, however, by an acknowledgement of some of the technical challenges around sound, bandwidth, and interface, suggesting a need for "systematic training and constant practice on feedback development" according to Hung (p. 100). The value of peer feedback during the composition of multimodal assignments is also recognized by O'Shea and Fawns in their discussion of collaborative connoisseurship in digital environments [48]. When students and their tutors "generally have a somewhat vague grasp of what represents academic quality within emerging multimodal practices or how to produce a multimodal product that conforms to assessment criteria and other requirements" (p. 226), there is considerable value in opportunities for activities and accompanying dialogue where expectations around what represents high quality work are made explicit. In a sense, the same benefits would surely be recognized by tutors and students concerned with more conventional essayistic or language-privileging assignments, however O'Shea and Fawns, supported by the testimonies of students they interviewed, correctly recognize a heightened level of uncertainty (and therefore need for dialogue around assessment) when meaning is represented across a richer range of communicational modes. 


\section{Case Study: Digital, Multimodal Dialogue as Feedback}

At this point, I would like to return to the scene from the beginning of the article. Through the use of a case study I wish to illustrate and explain how digital, multimodal dialogue can provide exciting and apt learning opportunities. Despite ambiguity around the methodological function of the case study [49], and the variety of ways that it manifests across research [50], the central purpose of the approach, according to Tobin [51], is "to help us notice and understand particular aspects of the human experience that are often overlooked or unexamined by other types of research" (p. 771). Thinking about education research in particular, the use of case studies has become particularly popular, according to Bailey [50], for instance, as a way of interrogating student experiences, attitudes towards technology, and a desire to improve teaching practices. This is a view echoed by Timmons and Cairns [52] when they argue that the case study approach "not only creates knowledge and understanding but also sets a standard for good teaching practices through two main means-development and implementation of policy, and gaining experience through exposure to a particular phenomenon" (p. 100). The value of the case study to the education researcher, according to Bailey, lies in the possibility of being able to connect specific events to wider patterns of practice, while also having the capacity to support the generation of theory. By narrowing the gaze to focus on particular phenomena or practices, the researcher foregoes the possibility of making the kind of broad generalizations that might emerge from large data sets (although Timmons and Cairns argue that a case study approach can support generalizability when used as part of a broader repertoire of methods, including surveys or interviews, for instance). Countering the accusation that the case study approach lacks the rigor of research undertaken across a wider range of data or contexts, Timmons and Cairns, along with Tobin, highlight rich description, nuance, and the depth of understanding that comes from narrowing the attention to what can, according to Bailey [50], be even a single example of practice. For the purpose of my own work, the case study approach enabled me to consider existing theoretical and empirical work around multimodality, feedback, and technology, with a view to raising questions about the potentialities and challenges of digitally-mediated multimodal dialogue around assessment. When digital education practices are heavily subject to context, I make no claim to generalizability (and neither would a case study approach be suited to this form of argumentation, according to Bailey). Instead, by considering vital themes from the literature through a small-scale study, I have sought to foreground some of the critical issues around multimodality, technology, and feedback, which might in turn be used to ask questions about the nature of pedagogic practices in other educational settings.

Graeme's Second Life assignment was completed within the Introduction to Digital Environments for Learning (IDEL) course, part of the MSc in Digital Education at the University of Edinburgh. The Digital Education program is delivered entirely online and attracts teachers working in primary, tertiary, and secondary education, as well as learning technologists, course designers, and other professionals in training roles. The program provides students with critical insights and practical skills relevant to the increasingly digital nature of education. An important part of the program culture sees students being encouraged to experiment with the representational form of their work through digital technologies; a commitment to multimodality is communicated through course handbooks, assessment criteria, and in conversation. The IDEL course took place across 12 weeks and was taught by a team of academic tutors, working with around four to five students each. This was a compulsory first course for all students on the Digital Education program. The fully online nature of the course and program meant that the cohort was made up of an international body of students. Although students were united in having previously participated in an undergraduate degree program, disciplinary background and current professional interest varied across the group. This included students who were undertaking their first experience of formal education since completing an undergraduate degree, to a number of the group who already held a doctorate. Similarly, students had enrolled on the Digital Education program for a range of reasons including an interest in exploring how academic practice was affected by the shift to the digital (as was the case for Katherine, whose work is discussed below) 
and a desire to take a critical approach to understanding how digital technologies might enhance their work in teaching or training (as in Graeme's case).

During the period described in this case study, the advertised purpose of the IDEL course was to offer a snapshot of the technological possibilities of digital education, while at the same time giving students an opportunity to explore some of the practical and theoretical issues concerned with learning in digital contexts. To complete the course, students had to participate in two assessment exercises: an ongoing reflective blog recording their thoughts and experiences in diary form, as well as a final assignment exploring a course theme of particular interest. In each case, students were encouraged to present their ideas in digital and multimodal formats relevant to the internet. The course itself was delivered across a range of digital environments including Skype, Second Life, and a discussion board within the Moodle learning management system, where students and staff would gather to discuss "presence," "sanctuary," "spaces", and other themes relevant to digital education. The most frequent interaction, however, took place within the reflective blog, hosted on the Elgg open source social networking platform, where students would produce around three posts per week sharing their understanding and experiences around the corresponding course topic. In my role as a tutor, I would read through the blogs for my five students before offering feedback in the form of encouragement, questions, and suggestions for further reading and reflections, communicated within the comments function under each post.

During week 4 of the IDEL course, students were invited to explore ideas around "body," "mind," and "community," while at the same time participating in a series of activities in the online virtual world of Second Life. This included assembling on the beach or gathering around the campfire for group tutorials with tutors and peers. Rather than responding to the corresponding blog posts in the conventional typed format, I instead delivered feedback "through" my own Second Life avatar, Jimmi. This involved using the SnapzPro screen capture software to create footage of Jimmi in a range of locations and poses in Second Life. I then edited the clips in iMovie software before adding a series of typed captions offering feedback in the form of comments, questions, and encouragement (Figure 2). Although the same feedback could have been more quickly and easily typed into the comments box within the Elgg blog, by taking a more experimental and richly multimodal approach, I wanted to encourage my tutees to move beyond printed language as the dominant representational mode within their work. During the early stages of the course, many of the reflective blogs were characterized by a heavy emphasis on words, sometimes preceded or interspersed with an illustrative image. When there is evidence that students are cautious about the disposable nature of the digital form [53] and that online platforms such as blogs can simply re-enforce the structure and linearity of more conventional print-based communication [54], I wished to reiterate that, within the exploratory setting of the IDEL course, approaches that might elsewhere be viewed as overly experimental or unconventional [55] could be a valid way of representing academic knowledge. In conversation with students, I explained my approach as "multimodal critical provocation": an attempt to challenge them to think about how the digital form is particularly suited to conveying knowledge in ways that are innovative, multimodal, and also scholarly.

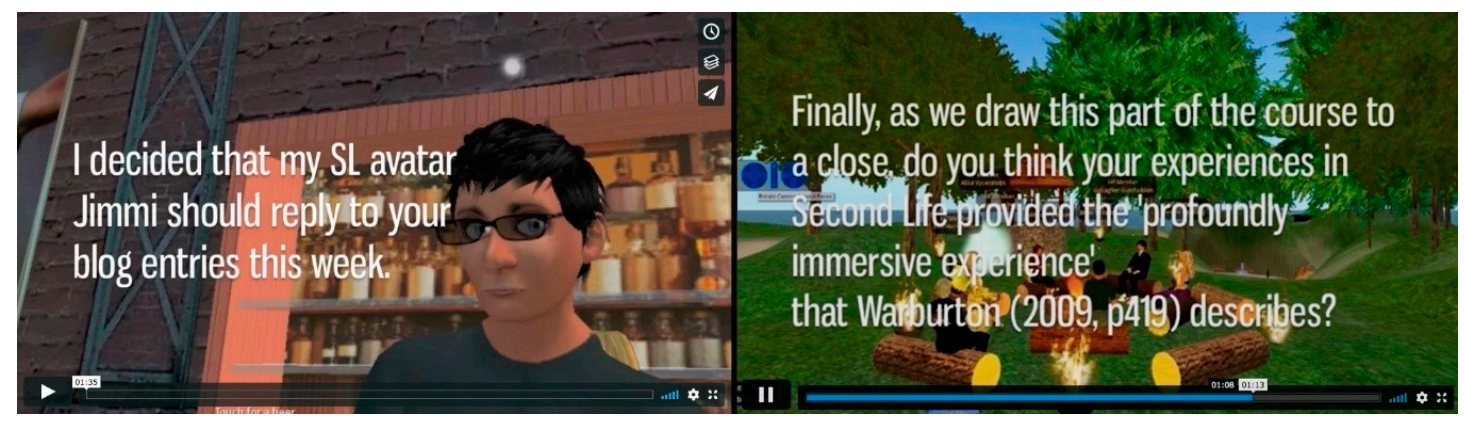

Figure 2. Stills from video feedback recorded in Second Life. Image by the author. 
In the weeks that followed, the reflective blogs became more richly multimodal as students began to place a greater emphasis on visual content alongside written reflection. Importantly, in the conversations I had with students about the representational form of their work, the emphasis was always about selecting and configuring modes in a way that would best convey their ideas, not about dispensing with printed language. To emphasize this point, at different times during our dialogue I directed students towards Kress's concepts of "aptness of mode" and "aptness of audience" [17]. In his discussion of the gains and losses around new forms of image-dominated representations of knowledge, Kress points towards the possibilities of orchestrating modes to best suit the knowledge to be conveyed and the perceived needs of the audience. Applied to the setting of the reflective blog and the IDEL course, this involved inviting students to think about selecting the representational form for their work that was most suited to the ideas they wished to communicate or demonstrate, whilst also meeting what they imagined to be my preferences and interests, as their audience (which included the expectations set out in the assessment criteria). With a background in musicology, Katherine took up this challenge by creating a music playlist in response to some of the preceding course themes. On other occasions, she used cartoons with short sentences combined with color, lines, and shapes to represent her ideas around the nature of digital authorship and presence in networked environments. Sustaining the emphasis on multimodal dialogue, my response in these instances came through a combination of music and image, including embedded videos and images edited in Photoshop that directly replied to Katherine's preceding ideas. What followed was a richly multimodal conversation played out through images and words that was simultaneously colorful and critical. For her final assignment, Katherine went on to produce a highly effective and richly multimodal web essay where she interspersed a typed commentary with music videos and images as she presented arguments around cyborg culture and posthumanism.

Similar multimodal exchanges took place in week 10 of the course as our attention turned to visuality, visual literacy, and the nature of knowledge construction within increasingly digital learning environments. Graeme approached the subject matter as an opportunity to enact the course content through the form of his work, as he described in a video blog post. Responding in kind, I produced my own screencast where I combined images and captioned citations with a spoken commentary. The multimodal dialogue between Graeme and myself would continue through to his final assignment where he used some of the same approaches to embed a videocast into the underwater classroom he had constructed in Second Life. Graeme's assignment was a particularly effective example of matching the representational form of his work to the ideas he wanted to convey; his arguments around immersion and presence in virtual worlds were advanced by inviting me to experience some of the same immersive and embodied experiences discussed in his commentary.

In terms of their representational form, these final assignments were considerably different from the predominantly language-based emphasis of earlier blog posts. At the time, Graeme and Katherine described how this digital multimodal dialogue excited and emboldened them towards these imaginative and highly successful approaches. These feelings were reiterated during recent email exchanges as I invited both students to look back upon their experiences of our exchanges and their subsequent composition of richly multimodal assignments. Where the literature around the introduction of multimodal assessment has sometimes highlighted uncertainty and reticence amongst students towards the digital form $[13,54,55]$, Graeme instead saw this "disruption" as a positive experience as he was challenged to playfully challenge conventional forms of authorship and meaning-making. Graeme and Katherine both highlighted the experiential value of being provoked into moving beyond the "essayistic" form in order to reflect on the changing nature of knowledge production within increasingly digitally-mediated learning environments. Also interesting was Katherine's belief that a major strength of the IDEL course culture was the expectation and opportunity for tutors to experiment with the representational form of their work (for instance, through multimodal dialogue), alongside their students. This serves to helpfully reiterate that the use of digital and richly multimodal assessment and feedback is a joint venture between students and tutors. Nevertheless, 
for all that our digital multimodal dialogue evidently contributed towards positive learning experiences and the composition of highly effective assignments, when educational activity is a performance of a broad range of social and material interests and opportunities [14], there is also a need to recognize other contributory factors [46]. This includes, for instance, the existence of a program culture that was supportive of multimodal representations of knowledge, as well as the time, technology, and talent at Graeme and Katherine's disposal. Again, this reifies Fenwick et al.'s position that a sociomaterial lens usefully draws attention to the way that educational practice is negotiated through a range of different opportunities, resources, and pressures, including but extending beyond those of the student and teacher [14].

\section{Discussion}

Having argued for the way that multimodal feedback can contribute towards the representation of academic knowledge in ways that are simultaneously imaginative, scholarly, and in-tune with our increasingly digitally-mediated world, it is important to consider how, for the teacher, this might be an invigorating or unsettling experience (or both). When case study research places considerable emphasis on the interpretation, action, and presence of the researcher, and has accordingly raised questions about the independence of the author reporting on the work [49], it is important to reflexively acknowledge how my own presence and interests will have shaped the experiences being reported here. Reflexivity is "the process of a continual internal dialogue and critical self-evaluation of researcher's positionality" [56] (p. 220), which acknowledges and explains how the researcher was implicated in a research activity [57]. When the researcher's presence, interests, and actions inevitably shape the responses and behavior of those communities or individuals being studied, reflexivity recognizes the impossibility of the researcher objectively separating herself from what is seen, heard, or performed, or what Hammersley describes as an Olympian perspective of viewing from the outside [58]. Drawing attention to my own status and interests within the research setting serves the purpose of highlighting the subjectivity of the work presented here, thereby supporting the reader's interpretation of the ideas being put forward.

My involvement with the IDEL course (previously as a student and then as a tutor) will have shaped my understanding and attitude in a way that would be different from an "outside" researcher or observer. Having completed the same coursework exercises as Graeme and Katherine a number of years previously, I had a level of empathy and deeper understanding of some of the challenges and opportunities they might experience, which will in turn have shaped the tone and content of our dialogue. My prior technical and critical experience of creating multimodal artefacts would not be shared by all teachers, and this also needs to be recognized in considering how the multimodal exchanges described here might translate to other settings. The particular technical and interpretive challenges of multimodal assessment might call for teachers who have honed their critical skills in a print-based tradition to seek out opportunities for professional development [59]. Extended to practices around feedback, a greater emphasis on multimodal dialogue, combined with a shifting representational landscape more generally [23], might reasonably highlight a need for some teachers to broaden their repertoire of skills to accommodate new approaches to meaning-making. On a related note, since the period described in this case study, the Digital Education program has gradually migrated away from Second Life to the increasingly popular educational space of Minecraft, prompting myself and other tutors to spend time with learning technologist colleagues as we oriented ourselves towards a new digital learning environment.

The multimodal dialogue described in the case studies was also supported by program culture: when a commitment to multimodal expression and experimentation with the digital form is made explicit through course handbooks and assessment criteria, the conditions are ripe for tutors to do likewise. It would be a different proposition, however, for the teacher who is uncertain over whether multimodal dialogue will align with the expectations of her students, colleagues, and external examiners, for instance. Furthermore, in light of the culture of benchmarking and performativity, 
the teacher might question how comfortably dialogue in the form of cartoons or images might sit with an institution-wide feedback strategy. Therefore, where the discourse around multimodality refers to acts of design and emphasizes choices around the selection and configuration of semiotic material in the communication of meaning [1], the theoretical implication of choice will not always translate to the assessment and feedback setting, where practices might be scrutinized for transparency, clarity, and consistency.

I would also draw attention to the way that my own research interests should be recognized when considering the work presented here. The generation of multimodal feedback initially proved to be a time-consuming exercise, thereby mirroring the experiences of Sadoux [36]. The preparation of each piece of Second Life video initially took around an hour, considerably longer than typing comments underneath a blog post. I felt the effort was justified by my wider critical interest in multimodal learning. I acknowledge, though, that in light of the competing pressures faced by academic staff, this might prove prohibitive for other teachers. Of course, multimodal dialogue encompasses a broad range of approaches and Campbell and Feldman [42] point towards the "efficiency" of providing feedback though video commentary while Sadoux describes how the initial investment in creating generic multimodal feedback through screencasts saved time in subsequent iterations of her course, on account of the same materials being used to support further cohorts of students. Although the personalized nature of student-tutor exchanges on the IDEL course rarely supported the recycling or use of generic feedback as Sadoux [36] describes, I have found the subsequent crafting of video feedback to be considerably easier and quicker on account of these earlier experiences.

Having argued that multimodal dialogue can enable students to recognize the validity and critical value of taking a multimodal approach in their work, I am not advocating the approaches described here to the exclusion of other more conventional (and possibly less time-consuming), language-based methods of feedback. When a convincing part of the rationale for placing greater emphasis on multimodality within assessment and feedback has been the need to align approaches with the evolving nature of meaning-making practices across society and education, we should recognize that a considerable amount of what takes places in schools, colleges, and universities remains deeply committed to the language in its various forms [53]. Drawing again on Kress' aptness of mode and audience [17], the challenge for the teacher is to design assessment tasks and craft feedback in ways that are best suited to the knowledge to be conveyed, alongside the interests of her students in that particular context. The use of multimodal dialogue is no more about dispensing with written feedback than multimodal assessment involves abandoning the essay; on the contrary, as Elola and Oskoz argue in their discussion of multimodal feedback in the foreign language classroom [45], we should instead consider using a repertoire of approaches.

It is also important to acknowledge that students involved in multimodal dialogue would certainly have a range of different stories to tell based upon their own interests, abilities, and circumstances. Therefore, while Graeme and Katherine spoke enthusiastically about the impact of our multimodal dialogue upon their learning, on other occasions when I have delivered seminars on the subject of multimodal composition and assessment, students have described how a perceived lack of time, technical skill, or creativity discouraged them from taking up the challenge of presenting their work in a multimodal form. Therefore, just as Mathisen [12] describes how some students may find a multimodal feedback approach less suited to their particular interests or circumstances, we are reminded that multimodal dialogue will not address all the challenges associated with feedback, as Philips et al. [46] acknowledge. When relatively little has been written specifically about student experiences of multimodal feedback, it can be helpful to look towards the wider literature around multimodal assessment in order to get a sense of the contrasting attitudes and emotions of learners. This varies from the enthusiasm of high school students thrilled at the opportunity to convey knowledge through informal and more familiar literacy practices [26] to a sense of skepticism around the "dubious" and "disposable" digital form [53] (p. 21), coupled with a reluctance to move beyond the tried-and-trusted linearity and language-based expression of essayistic approaches [55]. 


\section{Conclusions}

In this article, I have argued that approaching feedback as an ongoing dialogue presented in richly multimodal and digital form can support opportunities for learning that are imaginative, critical, and in-tune with our increasingly digitally-mediated society. When a teacher communicates with students in a richly multimodal way, she can demonstrate the academic validity of the form, potentially emboldening and encouraging her students towards approaches that might otherwise be perceived as unsuitable or risky, particularly in the assessment setting. Consistent with literature around multimodality and education more generally, I have argued that in our increasingly digitally-influenced learning environments and society, we need to consider when and how our approaches to feedback should pay greater attention towards opportunities for the multimodal representation of academic knowledge. This does not mean however that we dispense with print-based approaches to feedback; on the contrary, the question should always be about aligning feedback to the particular assessment and learning context, including the knowledge to be conveyed. At the same time, I have drawn attention to some of the practical and critical challenges that teachers might confront around multimodal dialogue, which includes recognizing that educational practice is always subject to a complex entanglement of social and material interests and opportunities that can support or sit in opposition to the feedback approaches described here. Without suggesting that the type of exchanges between myself and Graeme and Katherine would be suited to every student or situation, or that we should take the introduction of digitally-mediated feedback lightly, it is my hope that the article presented here encourages other teachers to boldly explore the exciting possibilities of digital, multimodal dialogue.

Funding: This article was prepared during my ESRC-funded Doctoral research at the University of Edinburgh.

Conflicts of Interest: The author declares no conflict of interest.

\section{References}

1. Kress, G.; Van Leeuwen, T. Multimodal Discourse: The Modes and Media of Contemporary Communication; Hodder Arnold: London, UK, 2001; ISBN 9780340662922.

2. Ong, W.J. Wired for Sound: Teaching, Communications, and Technological Culture. Coll. Engl. 1960, 21, 245-251. [CrossRef]

3. Jewitt, C. An introduction to multimodality. In The Routledge Handbook of Multimodal Analysis; Jewit, C., Ed.; Routledge: London, UK, 2009; pp. 14-27. ISBN 9780415667777.

4. Matthewman, S.; Blight, A.; Davies, C. What does multimodality mean for English? Creative tensions in teaching new texts and new literacies. Educ. Commun. Inf. 2004, 41, 153-176. [CrossRef]

5. Carpenter, R. Boundary negotiations: Electronic environments as interface. Comput. Compos. 2009, 26, 138-148. [CrossRef]

6. Kimber, K.; Wyatt-Smith, C. Secondary students' online use and creation of knowledge: Refocusing priorities for quality assessment and learning. Aust. J. Educ. Technol. 2010, 26, 607-625. [CrossRef]

7. Johnson, D.; Kress, G. Globalisation, Literacy and Society: Redesigning pedagogy and assessment. Assess. Educ. Princ. Policy. Pract. 2003, 10, 5-14. [CrossRef]

8. Jewitt, C. Technology, Literacy and Learning: A Multimodal Approach; Routledge: Abingdon, UK, 2006; ISBN 9780415345491.

9. Hatzipanagos, S.; Warburton, S. Feedback as dialogue: Exploring the links between formative assessment and social software in distance learning. Learn. Media Technol. 2009, 34, 45-59. [CrossRef]

10. Hounsell, D.; Xu, R.; Tai, C.-M. Balancing Assessment of and Assessment for Learning. (Scottish Enhancement Themes: Guides to Integrative Assessment, Nos. 2 \& 3) 2007. Gloucester: QAA. Available online: http:/ / www.enhancementthemes.ac.uk/docs/publications/guide-no-2-balancing-assessment-of-andassessment-for-learning.pdf?sfvrsn=16 (accessed on 21 May 2018).

11. Eistenstein, E.L. The Printing Press as An Agent of Change: Communications and Cultural Transformations in Early-Modern Europe (Volume 1); Cambridge University Press: Cambridge, UK, 1980; ISBN 0521299551. 
12. Mathisen, P. Video feedback in higher education-A contribution to improving the quality of written feedback. Univ. Nord. J. Digit. Lit. 2012, 7, 97-116. Available online: https://www.idunn.no/dk/2012/02/ video_feedback_in_higher_education_-_a_contribution_to_impr (accessed on 21 May 2018).

13. Bayne, S. What's the matter with 'technology enhanced learning'? Learn. Media Technol. 2014, 40, 5-20. [CrossRef]

14. Fenwick, T.; Edwards, R.; Sawchuk, P. Emerging Approaches to Educational Research: Tracing the Sociomaterial; Routledge: Abingdon, UK, 2011; ISBN 9780415570923.

15. Bhatt, I.; de Roock, R. Capturing the sociomateriality of digital literacy events. Res. Learn. Technol. $2013,21$. [CrossRef]

16. Johri, A. The socio-materiality of learning practices and implications for the field of learning technology. Res. Learn. Technol. 2011, 19, 207-217. [CrossRef]

17. Kress, G. Gains and losses: New forms of texts, knowledge, and learning. Comput. Compos. 2005, 22, 5-22. [CrossRef]

18. Kress, G.; Selander, S. Multimodal design, learning and cultures of recognition. Internet High. Educ. 2012, 15, 265-268. [CrossRef]

19. Newfield, D. Multimodality and children's participation in classrooms: Instances of research. Perspect. Educ. 2011, 29, 27-35.

20. Norris, S. Modal density and modal configurations. In The Routledge Handbook of Multimodal Analysis; Jewitt, C., Ed.; Routledge: London, UK, 2009; pp. 78-91. ISBN 9780415667777.

21. Mills, K.A. A review of the "Digital Turn" in the new literacy studies. Rev. Educ. Res. 2010, 80, 246-271. [CrossRef]

22. Miller, S.M.; Knips, M.A.; Goss, S. Changing the game of literature with authentic assessment: The promise of multimodal composing. Engl. J. 2013, 103, 88-94.

23. Archer, A. Clip-Art or design: Exploring the challenges of multimodal texts for writing centres in higher education. South. Afr. Linguist. Appl. Lang. Stud. 2011, 29, 387-399. [CrossRef]

24. Jewitt, C. Multimodality, "Reading", and "Writing" for the 21st Century. Discourse Stud. Cult. Polit. Educ. 2005, 26, 315-331. [CrossRef]

25. Krause, M.B. "A series of unfortunate events": The repercussions of print-literacy as the only literacy for talented boys. Gifted Child Today 2013, 36, 236-245. [CrossRef]

26. Newfield, D.; Andrew, D.; Stein, P.; Maungedzo, R. "No number can describe how good it was": Assessment issues in the multimodal classroom. Assess. Educ. Princ. Policy Pract. 2003, 10, 61-81. [CrossRef]

27. Kimber, K.; Wyatt-Smith, C. Rethinking quality assessment for 21st century learning: How students use and create knowledge online. In Proceedings of the International Association for Educational Assessment, Brisbane, Australia, 13-18 September 2009.

28. Boud, D.; Molloy, E. Rethinking models of feedback for learning: The challenge of design. Assess. Eval. High. Educ. 2013, 38, 698-712. [CrossRef]

29. Carless, D.; Boud, D. The development of student feedback literacy: Enabling uptake of feedback. Assess. Eval. High. Educ. 2018, 43, 1315-1325. [CrossRef]

30. Shute, V.J. Focus on formative feedback. Rev. Educ. Res. 2008, 78, 153-189. [CrossRef]

31. Nicol, D.; Macfarlane-Dick, D. Formative assessment and self-regulated learning: A model and seven principles of good feedback practice. Stud. High. Educ. 2006, 31, 199-218. [CrossRef]

32. Wang, S.L.; Wu, P.I. The role of feedback and self-efficacy on web-based learning: The social cognitive perspective. Comput. Educ. 2008, 51, 1589-1598. [CrossRef]

33. Bates, S.P.; Howie, K.; Murphy, A.S.J. The use of electronic voting systems in large lectures: Challenges and opportunities. New Dir. Teach. Phy. Sci. 2006, 2, 1-8. [CrossRef]

34. Ryan, B.J. Line up, line up: Using technology to align and enhance peer learning and assessment in a student centred foundation organic chemistry module. Chem. Educ. Res. Pract. 2013, 14, 229-238. [CrossRef]

35. Marriott, P.; Teoh, L.K. Using screencasts to enhance assessment feedback: Students' perceptions and preferences. Account. Educ. An Int. J. 2012, 21, 583-598. [CrossRef]

36. Sadoux, M. Generic online multimodal feedback on summative assessments. Invest. Univ. Teach. Learn. 2010, 6, 86-92.

37. Dawson, S.; Gasevic, D.; Siemens, G.; Joksimovic, S. Current State and Future Trends: A Citation Network Analysis of the Learning Analytics Field. In Proceedings of the International Conference on Learning Analytics and Knowledge, Indianapolis, IN, USA, 24-28 March 2014.

38. Gašević, D.; Dawson, S.; Siemens, G. Let's not forget: Learning analytics are about learning. TechTrends 2014, 59, 64-71. [CrossRef] 
39. Pardo, A. A feedback model for data-rich learning experiences. Assess. Eval. High. Educ. 2017, 43, 428-438. [CrossRef]

40. Pardo, A.; Jovanovic, J.V.; Dawson, S.; Gašević, D.; Mirriahi, N. Using learning analytics to scale the provision of personalised feedback. Br. J. Educ. Technol. 2017. [CrossRef]

41. Shute, V.; Ventura, M. Stealth Assessment: Measuring and Supporting Learning in Video Games; MIT Press: Cambridge, CA, USA, 2013; ISBN 978-0-262-51881-9.

42. Campbell, B.S.; Feldmann, A. The power of multimodal feedback. J. Curric. Teach. Learn. Leadersh. Educ. 2017, 2, 1 .

43. Nixon, R.S.; Smith, L.K. Teaching multiple modes of representation in middle-school science classroom: Impact on student learning and multimodal use. Sch. Sci. Math. 2015, 115. [CrossRef]

44. Keppell, M.; Carless, D. Learning-oriented assessment: A technology-based case study. Assess. Educ. Princ. Policy Pract. 2006, 13, 179-191. [CrossRef]

45. Elola, I.; Oskozz, A. Supporting second language writing using multimodal feedback. Foreign Lang. Ann. 2016, 49, 58-74. [CrossRef]

46. Phillips, M.; Henderson, M.; Ryan, T. Multimodal feedback is not always clearer, more useful or satisfying. In Show Me the Learning, Proceedings of ASCILITE 2016, Adelaide, Australia, 28-30 November 2016; Barker, S., Dawson, S., Pardo, A., Colvin, C., Eds.; ASCILITE: Adelaide, Australia, 2016; pp. 514-522.

47. Hung, S.T.A. Enhancing feedback provision through multimodal video technology. Comput. Educ. 2016, 98, 90-101. [CrossRef]

48. O'Shea, C.; Fawns, T. Disruptions and dialogues: Supporting collaborative connoisseurship in digital environments. In Advances and Innovations in University Assessment and Feedback; Kreber, C., Anderson, C., Entwistle, N., McArthur, J., Eds.; Edinburgh University Press: Edinburgh, UK, 2014; pp. 225-245. ISBN 9780748694556.

49. Verschuren, P. Case study as a research strategy: Some ambiguities and opportunities. Int. J. Soc. Res. Methodol. 2003, 6, 121-139. [CrossRef]

50. Bailey, L.E. Case study research. In Encyclopedia of Curriculum Studies; Kridel, C., Ed.; SAGE Publications: Thousand Oaks, CA, USA, 2010; pp. 104-105. ISBN 9781412958837.

51. Tobin, R. Quick start to case study research. In Encyclopedia of Case Study Research; Mills, A.J., Durepon, G., Wiebe, Eds.; SAGE Publications: Thousand Oaks, CA, USA, 2012; pp. 771-774. ISBN 9781412956703.

52. Timmons, V.; Cairns, E. Case study research in education. In Encyclopedia of Case Study Research; Mills, A.J., Durepon, G., Wiebe, E., Eds.; SAGE Publications: Thousand Oaks, CA, USA, 2012; pp. 100-102. ISBN 9781412956703.

53. Bayne, S. Temptation, trash and trust: The authorship and authority of digital texts. E-Learn. Digit. Media 2006, 3, 16-26. [CrossRef]

54. Lea, M.R.; Jones, S. Digital literacies in higher education: Exploring textual and technological practice. Stud. High. Educ. 2011, 36, 377-393. [CrossRef]

55. McKenna, C.; McAvinia, C. Difference and discontinuity-Making meaning through hypertexts. In Digital Difference: Perspectives on Online Learning; Land, R., Bayne, S., Eds.; Sense Publishers: Rotterdam, The Netherlands, 2011; pp. 45-60. ISBN 978-94-6091-579-6.

56. Berger, R. Now I see it, now I don't: Researcher's position and reflexivity in qualitative research. Qual. Res. 2013, 15, 219-234. [CrossRef]

57. Dowling, M. Reflexivity. In The SAGE Encyclopedia of Qualitative Research Methods; Given, L.M., Ed.; SAGE Publications: Thousand Oaks, CA, USA, 2012; p. 748. ISBN 9781412941631.

58. Hammersley, M. Reflexivity. In The SAGE Encyclopedia of Social Science Research Methods; Lewis-Beck, M.S., Bryman, A., Futing, T., Eds.; SAGE Publications: Thousand Oaks, CA, USA, 2011; pp. 934-935. ISBN 9780761923633.

59. Cumming, J.; Kimber, K.; Wyatt-Smith, C. Enacting policy: Curriculum and teacher conceptualisation of multimodal literacy and English in assessment and accountability. Engl. Aust. 2012, 47, 9-18.

(C) 2018 by the author. Licensee MDPI, Basel, Switzerland. This article is an open access article distributed under the terms and conditions of the Creative Commons Attribution (CC BY) license (http:/ / creativecommons.org/licenses/by/4.0/). 